\title{
Extracting Individual Respiratory Signatures from Combined Multi-Subject Mixtures with Varied Breathing Pattern Using Independent Component Analysis with the JADE Algorithm
}

\author{
Shekh M. M. Islam \\ dept. of Electrical Engineering \\ University of Hawaii at Manoa \\ Honolulu, HI, USA
}

\author{
Victor M. Lubecke \\ dept. of Electrical Engineeirng \\ University of Hawaii at Manoa \\ Honolulu, HI, USA
}

\begin{abstract}
Concurrent respiration monitoring of multiple subjects remains a challenge in microwave Doppler radar-based non-contact physiological sensing technology. Prior research using Independent component analysis with the JADE algorithm has been limited to the separation of respiratory signatures for normal breathing patterns. This paper investigates the feasibility of using the ICA-JADE algorithm with a 24-GHz phase comparison monopulse radar transceiver for separating respiratory signatures from combined mixtures of varied breathing patterns. Normal, fast, and slow breathing pattern variations likely to occur due to physiological activity, and emotional stress were used as a basis for assessing separation robustness. Experimental results showed efficacy for recognition of three different breathing patterns, and isolation of respiratory signatures with an accuracy of $100 \%$ for normal breathing, $92 \%$ for slow breathing, and $83.78 \%$ for fast breathing using ICA-JADE. Breathing pattern variations were observed to affect the signal-to-noise ratio through multiple mechanisms, decreasing with an increase in the number of breathing cycles and associated motion artifacts. Additionally, for removing motion artifacts of fast breathing pattern empirical mode decomposition (EMD) is employed, and for slow breathing pattern, increasing the breathing cycles helps to achieve an accuracy of $89.2 \%$ and $94.5 \%$ respectively.
\end{abstract}

Keywords- Remote sensing, separation, independent component analysis, breathing patterns.

\section{INTRODUCTION}

Remote sensing of human respiration using radar is gaining attention as breathing is one of the most important health metrics which can provide useful insights into human physiological states [1-2]. One of the potential applications of non-contact respiration sensing is home-based sleep monitoring especially for sleep apnea studies [3]. Moreover, respiratory radar has also shown efficacy in the recognition of individual people and remains under active investigation [3]. In home-based sleep monitoring or security system applications there is always a probability of the presence of multiple subjects in the radar system field of view [3-4]. This leads to combined mixtures of respiration patterns for which it is difficult to separate individual patterns [5]. Recently Lee et al. have demonstrated the efficacy of using FastIndependent component analysis (Fast ICA) for separating respiratory signatures from combined mixtures [5]. However, in their experiment, they used separate discrete receivers for each different subject which may be impractical for many scenarios. Fadel et al. have used frequency modulated continuous wave (FMCW) radar to isolate human subjects using wireless localization techniques [6]. In their experimental scenario equidistant subjects were not considered. Nosrati et al. have designed a dual-beam phased array radar for separating subjects by shifting the narrow beam in certain directions [7]. However, for this experiment, a minimum separation distance between subjects of 1 meter is required. In our prior attempt, we developed an intelligent SNR-based decision algorithm combining two different approaches ICA-JADE and direction of arrival (DOA) [8]. All reported work has been focused on isolating respiratory signatures when subjects have consistently normal breathing patterns [8]. Realistically, breathing patterns change due to daily activities or emotional stress [9]. Furthermore, during sleep apnea breathing occasionally stops. Thus, the efficacy of separation methods must be assessed for varied breathing patterns to understand their potential for practical application.

This paper reports on experiments for isolating respiratory signatures from combined mixtures of breathing patterns (normal, fast, and slow breathing) using a 24-GHz phase comparison Monopulse radar along with the ICA-JADE algorithm. It also evaluates the applicability of the ICA-JADE algorithm for extracting respiratory signatures from varied breathing patterns.

\section{THEORETICAL BACKGROUND}

\section{A. Respiration sensing for multiple subjects}

When multiple subjects are present in front of the sensor, each radar receives a combined mixture of breathing patterns from both subjects which makes it difficult to separate individual respiration signals [5-7]. Let us consider the transmitted signal from the radar transmitter is:

$$
S_{\text {transmitted }}=A_{c} \cos (\omega t+\theta)
$$

The reflected signal from one subject at the receiver is:

$$
S_{\text {reflected } 1}=A_{c}^{\prime} \cos \left[\omega t-\frac{4 \pi d}{\lambda}-\frac{4 \pi x(t)}{\lambda}+\theta(t)\right]
$$

Where, $A_{c}$ is the amplitude of the transmitted signal, $\lambda$ is the free space wavelength, $d$ is the distance of the radar from the subject, $\theta$ is the initial phase and $\theta(t)$ is the phase change of the transmitted signal. As there are two subjects present the combined mixture of breathing patterns is received. The inphase signal received is: $B_{\text {Icombined }}=B_{I 1}(t)+B_{I 2}(t)$ where, $B_{I 1}(t)$ is the in-phase signal from subject 1 and $B_{I 2}(t)$ is the in-phase signal from subject 2 . Similarly, in a quadrature 
receiver, the combined signal is $B_{Q \text { combined }}=B_{Q 1}(t)+$ $B_{Q 2}(t)$.

\section{B. ICA with the JADE Algorithm}

ICA is a powerful statistical method that recovers pure source signals from combined mixtures [10-12]. Several different algorithms are described in the literature, where fastICA, Infomax, and Joint approximate diagonalization of Eigenmatrices (JADE) are the most popular [11]. The JADE algorithm was chosen as it is a matrix computation based algorithm where diagonalization of eigenmatrices is performed [11]. It is a kurtosis based computational technique that finds the maximum non-gaussianity or independence of signals by finding their kurtosis [10].

\section{EXPERIMENTAL SETUP}

\section{A. Experimental Setup}

In this experiment, we used a $24-\mathrm{GHz} \mathrm{K}-\mathrm{MC} 4$ monopulse radar transceiver with four channels $\left(I_{1}, Q_{1}, I_{2}\right.$ and $\left.Q_{2}\right)$. These four channels were ac-coupled to four low-noise amplifiers (LNAs) (SR560) with a cut-off frequency of $30 \mathrm{~Hz}$ and a gain of 200. The four output channels were connected to a DAQ (National Instrument) and a customized LABVIEW interface was used to capture all signals. Chest belts were used for reference measurements of individual respiration patterns. Participating subjects were asked to exhibit three different breathing patterns in front of the radar system. These patterns were normal, fast, and slow breathing. Two subjects were present in front of the system at a distance of 0.5 meters with an angular discrimination limit of 0.4 meters. Fig. 1 illustrates the presence of two subjects in front of the $24-\mathrm{GHz}$ radar system in a millimeter-wave anechoic chamber. All experiments were performed in a controlled environment and participants were seated. The experimental procedures involving human subjects were approved by an Institutional Review Board.

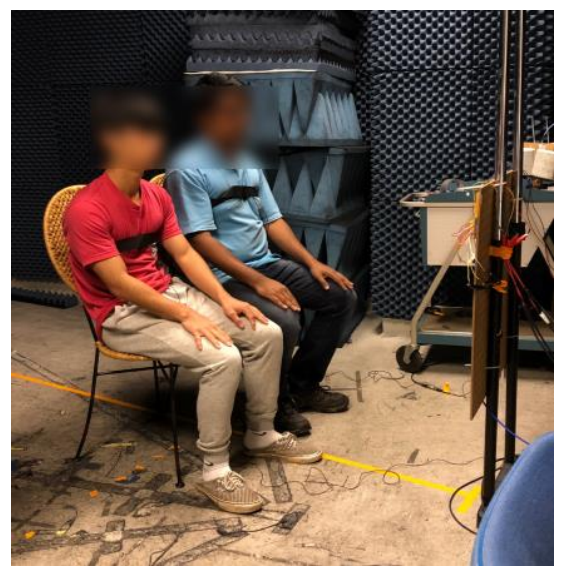

Fig. 1. Presence of multiple subjects in front of quadrature radar transceiver system in a millimeter wave anechoic chamber. A combined mixture of reflected signals returned to the radar from two subjects.

\section{B. Source separation process flow}

After collecting combined reflection data for varied breathing patterns, the data was linearly demodulated and mixed with random noise in a mixing matrix. After applying the JADE algorithm, independent respiratory signatures were separated from the combined mixtures. The mean square error and cross-correlation coefficient of the separated sources were

This work was supported in part by the National Science Foundation (NSF) under Grant IIP-1831303 and Grant IIS-1915738. calculated concerning a chest belt measured reference respiration pattern. A Fast Fourier transform (FFT) of the separated source and reference chest belt respiration pattern was performed for calculating the accuracy of the method.

\section{RESULTS}

Fig. 2 illustrates the radar captured respiration patterns for a combined mixture of normal and fast breathing patterns for two subjects in front of the radar system. It was observed that when both subjects exhibited faster-breathing patterns breathing depth increased. Fig. 2 illustrates the three different combined mixtures of respiratory patterns and corresponding I/Q plots. The linearly demodulated signals were mixed with random noise and a mixing matrix, and the output was used in the JADE algorithm to isolate independent respiratory patterns from the combined mixtures of the signals. The
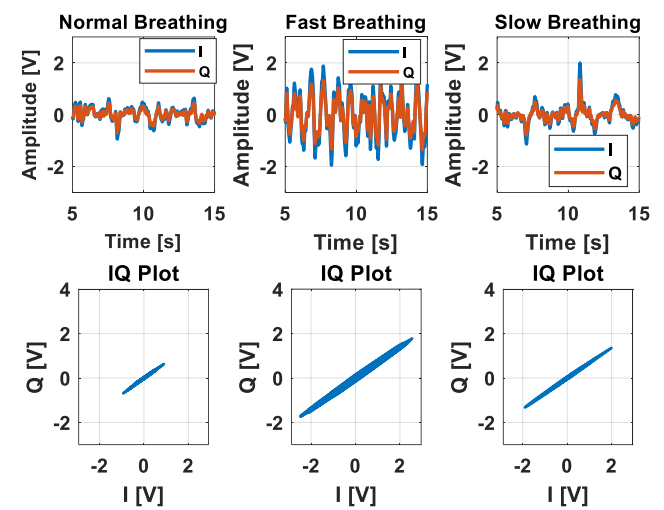

Fig. 2. Radar captured respiratory patterns for combined mixtures where subjects exhibited normal, fast and slow breathing patterns. Fast breathing pattern has a higher number of cycles and I/Q plot indicates a less clearly retraced arc pattern.

combined and separated sources for normal and fast breathing are shown in Fig. 3 where it is clear that the separated source for normal breathing closely correlates with the chest belt reference. On the other hand, for the fast breathing case, the separated source does not correlate as closely. The mean square error and cross-correlation coefficient of the separated source signals was calculated to the reference measurement. It was observed that, when subjects exhibit fast breathing patterns, they had difficulty breathing at this rate without creating extraneous motion artifacts [8]. Extraneous motion artifacts also appear in the I/Q plots where averaging was affected by the increased number of breath cycles per window. This also affected the phase measurement accuracy for the irregular signal due to the lower signal to noise ratio for this situation. For slow breathing, a distortion occurs due to the LNA's ac coupling which resulted in a larger error to the reference measurement. The cross-correlation coefficients of the separated source with the corresponding chest belt signal for normal, slow, and fast breathing patterns were 0.9971, 0.9335 , and 0.8634 respectively. An FFT was also used to compare rates for the radar and reference measurements. Fig. 3 illustrates the separated source for normal breathing and fast breathing and an FFT error analysis for the separated signal and chest belt signal. For slow breathing patterns, increasing the 20 -second window size with a 60 -second data set helps to achieve an accuracy of $94.5 \%$. For removing the motion artifacts of fast breathing pattern EMD method is employed to extract intrinsic mode function (IMF) from the filtered signal [13-14]. Fig. 4 below illustrates the extracted IMF signals 
from the radar-captured fast breathing patterns. The desired IMF
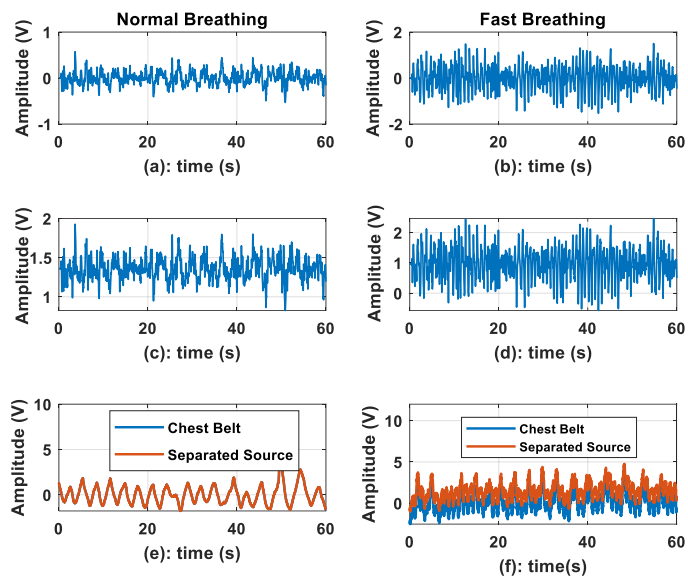

Fig. 3. (a) Linearly demodulated signal for normal breathing pattern (c) combined mixture of breathing pattern (e) separated source from combined mixture for normal breathing pattern. Similarly (b), (d) and (f) are for fast breathing pattern.

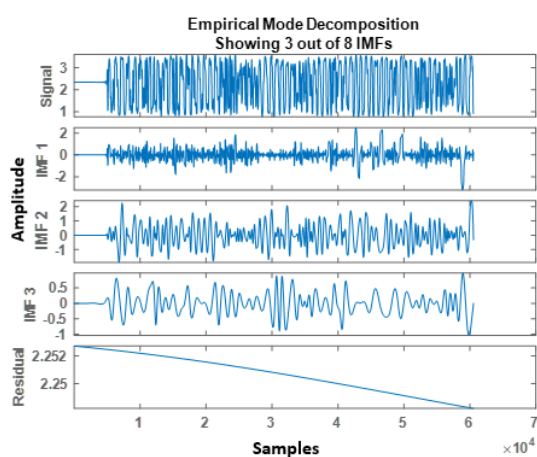

(a)

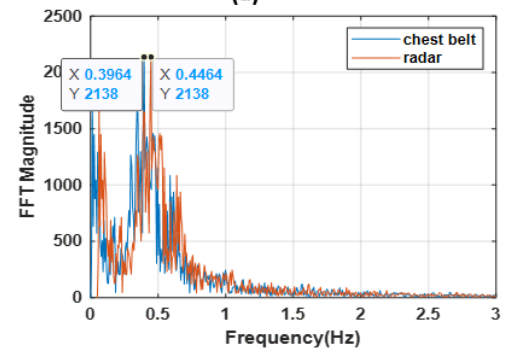

(b)

Fig. 4. (a) Empirical mode decomposition of the fast breathing pattern to remove the motion artifacts. IMF1, IMF2, IMF3 and residual is shown above. (b) FFT of the fast breathing pattern (blue line indicates chest belt signal and red line indicates radar captured signal).

TABLE I

SUMMARY OF ACCURACY FOR BREATHING PATTERNS

\begin{tabular}{|c|c|c|c|c|}
\hline $\begin{array}{c}\text { Breathing } \\
\text { Type }\end{array}$ & $\begin{array}{c}\text { Captured } \\
\text { Breathing } \\
\text { Rate (Hz) }\end{array}$ & $\begin{array}{c}\text { MSE } \\
\text { with } \\
\text { chest } \\
\text { belt } \\
\text { pattern }\end{array}$ & $\begin{array}{c}\text { Cross } \\
\text { correlation } \\
\text { coefficient }\end{array}$ & $\begin{array}{c}\text { Accuracy } \\
(\%)\end{array}$ \\
\hline Normal & .2 & $0.05 \%$ & .99 & $100 \%$ \\
\hline Slow & .11 & $5.23 \%$ & .93 & $94.5 \%$ \\
\hline Fast & .44 & $9.5 \%$ & .89 & $89.2 \%$ \\
\hline
\end{tabular}

was selected based on the SNR and then FFT is performed. Fig. 4 (b) shows the chest belt breathing rate is around $.4 \mathrm{~Hz}$ and radar extracted breathing rate is $.45 \mathrm{~Hz}$ which provides an accuracy of $89.2 \%$. Table-I summarizes the performance of the JADE algorithm for extracting breathing rates from combined mixtures for three different breathing patterns (normal, fast, and slow).

\section{CONCLUSION}

In this paper, we investigated the feasibility of separating respiratory signatures for combined mixtures of varied breathing patterns using the ICA-JADE algorithm. While the study indicated a usefully accurate measurement of separated signals, the results were affected by the decrease in SNR for the extreme breathing cases. This study illustrates the challenges associated with tracking respiratory patterns which significantly deviate from normal and its associated solutions (i.e. EMD). The proposed system has several potential applications especially for in-home sleep monitoring, automotive in-cabin monitoring, and security/surveillance applications.

\section{REFERENCES}

[1] J. C. Lin, "Noninvasive microwave measurement of respiration", Proc. IEEE, vol. 63, no. 10, pp. 1530, Oct. 1975.

[2] S. M. M. Islam, O. Borić-Lubecke, Y. Zheng, and V. M. Lubecke, "Radar-Based Non-Contact Continuous Identity Authentication," Remote Sensing, vol. 12, no. 14, p. 2279, Jul. 2020, doi: 10.3390/rs 12142279 .

[3] C. Li, V. M. Lubecke, O. Boric-Lubecke, and J. Lin, "A Review on Recent Advances in Doppler Radar Sensor for Non-Contact Healthcare Monitoring", in IEEE Transaction on Microwave Theory and Techn., vol. 61, pp. 2046-2060, May 2013.

[4] M. Baboli, A. Singh, B. Soll, O. Boric-Lubecke, and V. M. Lubecke, "Good Night: Sleep Monitoring Using a Physiological Radar Monitoring System Integrated with a Polysomnography System”. IEEE Microwave Magazine, vol. 16, no. 6 July 2015, pp. 34-41.

[5] S. M. M. Islam, E. Yavari, A. Rahman, V. M. Lubecke, and O. BoricLubecke, "Direction of Arrival Estimation of Physiological Signals of Multiple Subjects Using Phase Comparison Monopulse Radar," in 2018 Asia-Pacific Microwave Conference (APMC), Kyoto, 2018.

[6] S. Yue, H. He, H. Wang, H. Rahul, and D. Katabai, "Extracting MultiPerson Respiration from Entangled RF Signals," in Proceedings of the ACM on Interactive, Mobile, Wearable and Ubiquitous Technologies, vol. 1, no. 1, June 2018 .

[7] S. M. M. Islam, E. Yavari, A. Rahman, V. M. Lubecke, and O. BoricLubecke, "Multiple Subject Respiratory Pattern Recognition and Estimation of Direction of Arrival using Phase-Comparison Monopulse Radar," in 2019 IEEE Radio and Wireless Symposium (RWS), Orlando, FL, USA, 2019, pp. 1-4.

[8] S. M. M. Islam, O. Boric-Lubecke, and V. M. Lubecke, "Concurrent Respiration Monitoring of Multiple Subjects by Phase-Comparison Monopulse Radar Using Independent Component Analysis (ICA) with JADE Algorithm and Direction of Arrival (DOA)," in IEEE Access, vol. 8 (1), pp. 73558-73569, April 2020.

[9] S. M. Sherman, "Monopulse Principle and Techniques", Artech House: Boston, MA, USA, 1984.

[10] https: ://www.rfbeam.ch/product?id=18

[11] R. D. Degrot., "The Constrained MUSIC Problem", IEEE Transaction on Signal Processing, vol. 41 (3), pp. 1445-1449.

[12] O. R. Schmidt, "Multiple Emitter location and Signal Parameter Estimation", IEEE Transaction on Antenna Propagation, vol. 34(3), March 1986, pp. 276-280.

[13] M. Sedlacek and M. Krumphole, "Digital Measurement of Phase Difference- A Comparative Study of DSP Algorithms," Metrology and Measurement Systems, Vol. 12 (4), pp. 427-448, 2005.

[14] J. D. Capello, D. Strenski, "A practical measure of FPGA floating point acceleration for High Performance Computing", IEEE Int. Conf Application Specific Systems, Architecture and Processors, pp. 160167, Jun 2013. 